\title{
BMJ Open Patient experience of hospital care in China: major findings from the Chinese Patient Experience Questionnaire Survey (2016-2018)
}

\author{
Guangyu Hu, ${ }^{\oplus, 2}$ Yin Chen, ${ }^{3}$ Qiannan Liu, ${ }^{\oplus}$ Shichao Wu, ${ }^{2}$ Jing Guo, ${ }^{2}$ Shiyang Liu, ${ }^{2}$ \\ Zijuan Wang, ${ }^{2}$ Pengyu Zhao, ${ }^{2}$ Jing Sun, ${ }^{\circ}{ }^{2}$ Linlin Hu, ${ }^{2}$ Huixuan Zhou, ${ }^{2}$ Li Luo, ${ }^{5}$ \\ Ying Mao, ${ }^{6}$ Jack Needleman, ${ }^{7}$ Jing Ma, ${ }^{8}$ Yuanli Liu ${ }^{\circ}{ }^{2}$
}

To cite: Hu G, Chen Y, Liu Q, et al. Patient experience of hospital care in China: major findings from the Chinese Patient Experience Questionnaire Survey (2016-2018). BMJ Open 2019;9:e031615. doi:10.1136/ bmjopen-2019-031615

- Prepublication history and additional material for this paper are available online. To view please visit the journal (http:// dx.doi.org/10.1136/bmjopen2019-031615).

Received 13 May 2019 Revised 20 August 2019 Accepted 21 August 2019

D) Check for updates

(C) Author(s) (or their employer(s)) 2019. Re-use permitted under CC BY-NC. No commercial re-use. See rights and permissions. Published by BMJ.

For numbered affiliations see end of article.

Correspondence to Professor Jing Ma; jing_ma@harvardpilgrim.org

Professor Yuanli Liu; liuyl_fpo@126.com

\section{ABSTRACT}

Objectives China launched the National Healthcare Improvement Initiative (NHII) in 2015 to improve patient experiences in healthcare. This study aimed to generate evidence of hospital care quality from the patients' perspective.

Design This nationwide cross-sectional study interviewed participants from 31 provinces, municipalities and autonomous regions across China.

Setting A total of 117 tertiary hospitals in mainland China. Participants 48422 responses from outpatients and 35 957 responses from inpatients were included in this study. Primary outcome measure The scores of six predefined domains in the Chinese Patient Experience Questionnaire, five of which were designed to reflect specific dimensions of care, and one of which indicated the overall rating. Results More than $80 \%$ of the respondents viewed their care experiences as positive. The NHII seems to have had a positive impact, as indicated by the steady, although unremarkable, increase in the patient experience scores over the 2016-2018 period. The Chinese patients generally reported a positive experience with the clinical aspects of care, but reported a less positive experience with the environmental, interpersonal and social services aspects of care. The institutional factors, including region and type of hospital, and personal factors, such as gender, age, education and occupation, were factors affecting the patient experience in China. Humanistic care was the aspect of care with the greatest association with the overall patient experience rating in both the outpatient and inpatient settings.

Conclusions The national survey indicated an overall positive patient perspective of care in China. Older age, higher education level and formal employment status were found to be correlated with positive care experiences, as were higher levels of economic development of the region, a more generous insurance benefits package and a higher degree of coordinated care. The interpersonal-related initiatives had substantial roles in the improvement of the patient experience. In the regions where farmers and users of traditional Chinese medicine services constitute a greater proportion of the population, improvement of patient experiences for these groups deserves special policy attention.
Strengths and limitations of this study

- This study presented an overview of patient perspectives of care in China tertiary hospitals based on a large national survey.

- The Chinese Patient Experience Questionnaire (CPEQ), as a validated survey instrument, made it possible to compare the patient experience across domains indicating different attributes of the care that patients received.

- Aspects of care for further improvement were highlighted by data visualisation based on the pooled data analysis of the two rounds of national surveys.

- In the absence of weighting, the non-response bias might affect the reliability of the measured results.

- The results are presented without case-mix adjustment in the analysis, and caution should be used in their interpretation. A patient-mix adjustment model for $\mathrm{CPEQ}$ analysis should be considered in future studies.

\section{INTRODUCTION}

While universal coverage of basic social health insurance schemes has been achieved and affordability of healthcare services has improved, there has been a growing concern among the Chinese patients about the clinical, interpersonal and amenities aspects of their healthcare experience. ${ }^{2}$ Reportedly, the doctor-patient relationship had been worsening, and the incidence of violence against healthcare providers had been on the rise in China in recent years. ${ }^{3}$ Patient concerns about health service delivery in the public hospitals is one of the least understood aspects of the new round of health system reform in China that was launched in 2009.

Patients' views are essential to achieving high-quality care, and the patient experience has been considered to be a critical measure. ${ }^{4}$ Several definitions of the patient experience exist, and they commonly highlight 
occurrences and events involving patients across the continuum of care as well as patient feedback. ${ }^{56}$ As another commonly used measure to obtain knowledge from the perspective of patient, patient satisfaction is often misidentified and interchangeably used as patient experience; however, existing literature on the former is challenged by the absence of a universally accepted conceptual basis and measurement tool. ${ }^{78}$ On the other hand, the measurement of satisfaction is influenced by patient expectation, which could be insensitive in tracking the performance of providers over time ${ }^{49}$; thus, the patient experience has been increasingly monitored by researchers and regulators in North America ${ }^{10-12}$ and Europe $^{13-17}$ during the past decade. The National Health Service (NHS) in England led the way internationally in mandating annual national patient experience surveys instituted in $2001 .{ }^{18}$ The NHS patient survey programme has become a regulatory feature of the NHS. The Hospital Consumer Assessment of Healthcare Providers and Systems (HCAHPS) survey in America was implemented nationally in $2006,{ }^{12}$ and the measures of the patient experience were linked to actual payments for performance as a vital aspect of hospital quality since 2012. ${ }^{19}$ By contrast, China still lacks knowledge on this issue, and concerns about the representative and public reporting of patient experience survey results were inevitable.

As the healthcare quality improvement collaboratives proliferated internationally, ${ }^{20}$ the National Health Commission of the People's Republic of China posted an announcement implementing the National Healthcare Improvement Initiative (NHII) (initiative) in January $2015 .^{21}$ This initiative was a response to the central government's call for action on the progress of public hospital reforms and the people's issues of most concern regarding the healthcare delivery system. The announcement and the implementation strategy that followed over a 3-year period (2015-2017) signalled one of the world's largest initiatives intended to enhance the quality of care services, encompassing all Chinese public healthcare facilities, with the overall goal of improving the patient experience on a national level. ${ }^{22}$ Given this important national policy in China and the experiences from other countries, Peking Union Medical College (PUMC) School of Public Health addressed opportunities to design and conduct the China Healthcare Improvement Evaluation Survey (CHIES) as an independent third-party assessment of the initiative. While several studies have collected information on the patient experience and some regional surveys were conducted, ${ }^{23-28}$ prior to the CHIES, there was no national standard for collecting or publicly reporting data on patients' perspectives of care that would enable valid comparisons to be made across hospitals in China.

We aimed to generate evidence of the quality of care from the patient perspective in tertiary hospitals of different categories. Making use of data generated from the Chinese Patient Experience Questionnaire (CPEQ) survey as a part of the CHIES conducted in 2016 and 2018, we present the overview of the patient experience in China and an assessment of the association between patient characteristics and the experience of the healthcare they received.

\section{METHODS}

\section{Study design}

The CPEQ was developed by the PUMC to be a standardised survey instrument of the patient experience for the outpatient and inpatient settings simultaneously and in the Chinese context, with individual parts for ambulatory care (CPEQ-A) and inpatient care (CPEQ-I) (online supplementary appendix 1 ) ${ }^{29}$ We set up regional steering committees in 31 provinces, municipalities and autonomous regions (PMAs), consisting of representatives from regional hospital associations, the centre for quality control, local hospital administrators and medical school faculty, to administer the local survey. The CPEQ survey was approved by the institutional review board of the PUMC and conducted in both January 2016 (baseline) and January 2018 (end of the 3-year initiative). All respondents provided their oral consent to participate in the survey before the interview.

\section{Setting and participants}

Hospitals

For each PMA, we selected the provincial general hospital, the provincial traditional Chinese medicine hospital (TCM), and the provincial obstetrics and gynaecology hospital $(\mathrm{OB} / \mathrm{Gyn})$ plus teaching hospitals in that province administered by the national health authority. Altogether, the 136 hospitals thus selected constitute China's national and regional referral centres, which provide a disproportionately large share of healthcare services in China. The 136 tertiary hospitals across 31 PMAs included 57 general hospitals, 31 TCM hospitals and 29 OB/Gyn hospitals, while 19 specialised hospitals were excluded in this research due to the complicated case-mix of these hospitals.

\section{Patients}

Patients aged 15 years or older were included in this study, with additional inclusion criteria for the CPEQ-A: outpatients who finished and paid for their medical consultation, and for CPEQ-I: inpatients who received a discharge notice. Patients in the emergency or psychiatry department were excluded.

With the help of volunteers from each sampled hospital, we recruited patients to participate in the survey anonymously. The CPEQ-A was administered to outpatients in the drug dispensing area, where the outpatient usually finishes the visit. The CPEQ-I was administered to inpatients in the ward that would discharge the patient from hospital on the day they were interviewed. The survey was conducted by independent interviewers who were equipped with the online survey system on mobile devices, and respondents used the device to complete the online survey with help from the interviewer. For the 
elderly respondents, we also provided a paper-based questionnaire for their use.

\section{Outcome measures}

The primary outcomes measured by this study were the scores assigned to the survey questions in six predefined domains on the CPEQ-A and CPEQ-I, five of which were designed to reflect a specific dimension of care and one of which indicated an overall rating. The score of each domain was calculated from composite ratings by averaging the responses to each individual item within the domain. ${ }^{30}$ Each patient experience item in the CPEQ displays a description of specific service experiences in the hospital, and responses were explicitly designed to probe the degree of conformity of the description (possible responses: strongly agree, agree, neither agree nor disagree, disagree and strongly disagree). Patients were asked to rate their perceived experience on a scale of 1 (strongly disagree) to 5 (strongly agree). Detailed item specifications and the corresponding domains are shown in the online supplementary appendix 2 . We also calculated each score of the 23 patient experience items in the CPEQ-A and the 19 patient experience items in the CPEQ-I across the different types of hospitals. We predefined the 'strongly agree' and 'agree' responses to the item of description of specific service experiences to indicate a positive experience. Thus, a high score of the item reflects a better patient experience. We obtained information on the characteristics of the survey respondents, including gender, age, educational level, employment and health insurance coverage, as potential correlates. To assess the extent of organised healthcare delivery in China, we also asked the ambulatory care patients whether his/her clinical visit was prescheduled, and we asked the hospitalised patients if the hospital admission was performed through a referral system.

\section{Quality control}

The quality control precautions of the survey included a web-based data collection system design, standardised interviewer training, and on-site inspections. First, we developed an online survey system based on mobile devices (self-adaption for both smartphones and tablets) for the patient interview, which was designed to perform logic checks to identify illogical response and determine completeness of the records. A predefined coding system was embedded using hospital ID, questionnaire category, data acquisition time, interviewer number and respondent sequence number as a composite key index for identification of all patient records. When eligible patients were available to meet the sample size for each hospital, the interviewer would be alerted by the system. Encrypted data transmission was employed to ensure data security during the web-based transfer process. Second, the CPEQ survey was administered in the hospital by trained interviewers enrolled by the local steering committees. We organised survey training for key personnel from the 31 regional steering committees at a 2-day workshop organised by PUMC before each round of the survey. Meanwhile, a package of standardised training material was prepared by PUMC and provided to the regional steering committees for training their local fieldwork interviewers to ensure that the survey was administered uniformly. Third, coordinators from PUMC delivered on-site training with each local steering committee and acting as on-site inspectors for the survey to ensure data quality and maintain the projected timeframe.

\section{Sample size}

According to our pilot study results from the field test in Beijing, the mean score of the composite measure of the overall rating of the patient experience was 4.5 for both outpatients and inpatients. We aimed to make reliable estimates of patient experience scores for both the ambulatory care and inpatient care lines within each hospital. Therefore, we calculated the sample sizes based on the upper limit of the daily outpatient visits (10000 patients per day) and discharged inpatients (500 patients per day) in the sample hospitals. As a result, 206 and 148 respondents would be required to detect a mean score of 4.5 in the composite measure in the overall rating of the patient experience for ambulatory care and inpatient care in a hospital, respectively, with a $1.5 \%$ estimate range, $5 \%$ significance level and SD of 0.5. We thus set the targeted sample size at a minimum of 200 outpatients and 150 inpatients for each hospital.

\section{Statistical analysis}

The 117 hospitals were disaggregated by geographic locations: east, central and west (online supplementary appendix 3). The respondents were divided into three age groups: under $30,30-59$ and $\geq 60$ years. We described the characteristics of patient samples by using counts and proportions. We calculated the mean scores and 95\% CIs for each of the six predefined patient experience domain measures within all hospitals and hospital subgroups for both 2016 and 2018. We used the Kruskal-Wallis test to compare the patient experience scores between baseline and terminal surveys and across subgroups and specific domains. We applied a heatmap to visualise the CPEQ score performance of all the individual items over the different hospital categories from all pooled data of the two survey rounds, with the greenest colour indicating the best performance and reddest indicating the worst. To determine the specific characteristics associated with the overall rating of the patient experience in subgroups, we constructed two multivariable linear regression models that adjusted for all the other characteristics that might be potential confounders; the dependent variables were the overall rating of the patient experience on the CPEQ-A and CPEQ-I. To assess the independent relationship between performance in separate domains and the overall rating of the patient experience for outpatient and inpatient settings stratified by hospital categories, we constructed multivariable linear regression models to adjust for geographical locations, gender, age, education, 


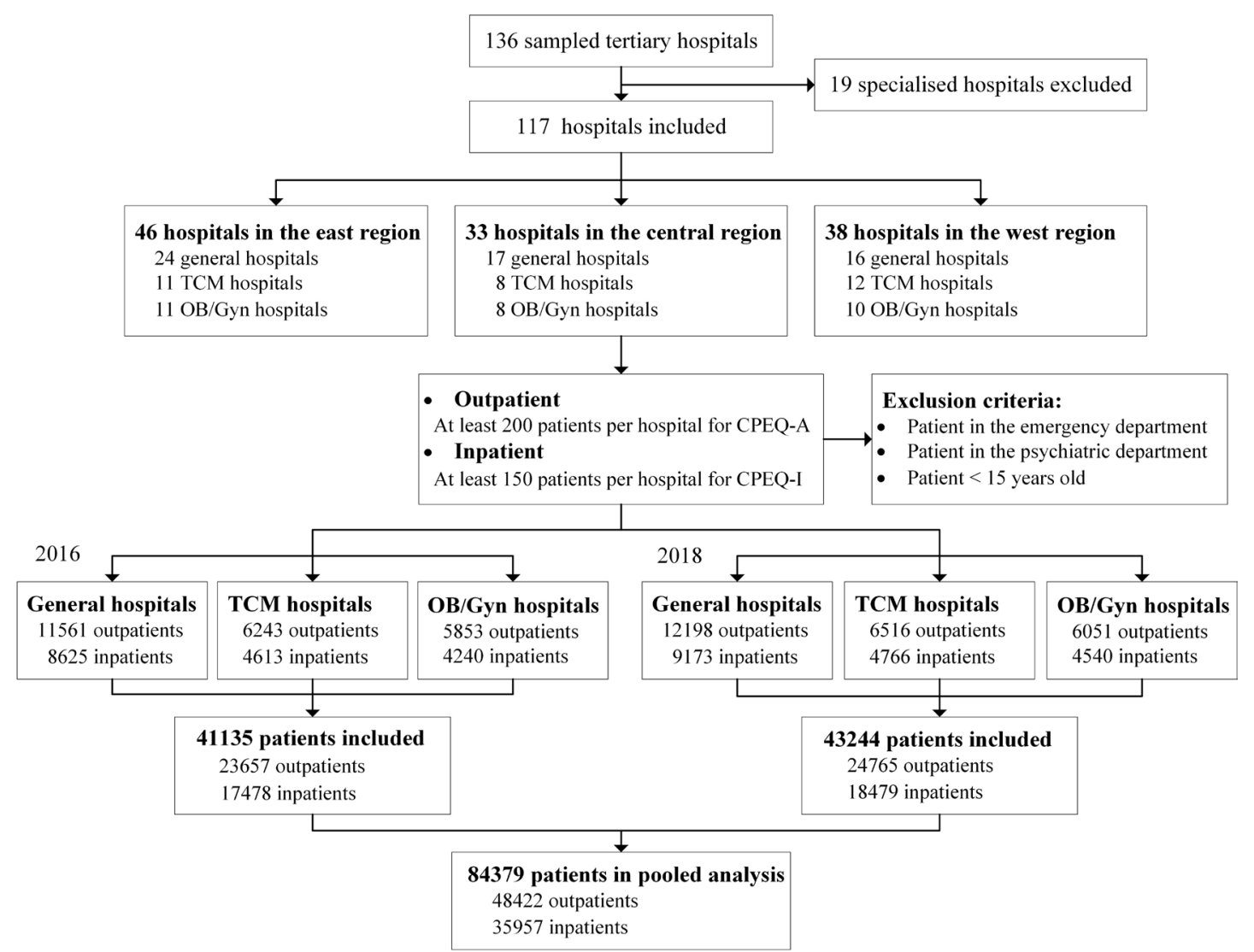

Figure 1 Flowchart of study participant selection in the CPEQ survey. CPEQ, Chinese Patient Experience Questionnaire; TCM,traditional Chinese medicine hospital; OB/Gyn,obstetrics and gynaecology hospital.

employment and health insurance coverage. We considered a $p$ value of $<0.05$ to be significant and used the $R$ stats package V.3.4.3 for all the data analyses.

\section{Participant and public involvement}

We conducted focus groups and cognitive interviews with patients from representative tertiary hospitals in Chengdu, Shanghai and Xi'an to draft the initial questionnaire. A 2-day workshop was organised in PUMC in January 2016, and experts from 31 PMAs in mainland China attended the meeting to discuss the provisional draft of the questionnaire. No patients and public were directly involved in the design and conception of the study nor the analysis or interpretation of the results. The results of the survey were disseminated through the publication of publicly accessible survey reports and presented at a National Conference on Healthcare Improvement in 2018.

\section{RESULTS}

\section{Characteristics of the sample}

From the 117 sampled hospitals, 48422 ambulatory care users completed the two rounds of surveys (23 657 in 2016 and 24765 in 2018), with an overall response rate of $50.3 \%$ in 2016 and $73.2 \%$ in 2018. In total, 35957 hospitalised patients completed the two rounds of surveys (17
478 in 2016 and 18479 in 2018), with an overall response rate of $90.0 \%$ in 2016 and $88.2 \%$ in 2018 (figure 1).

Descriptive statistics for the baseline and terminal survey samples are shown in table 1 . The outpatients and inpatients sample sizes were nearly equal in the two survey rounds, and both outpatient and inpatient respondents were comparable for most socioeconomic variables. The large proportion of female respondents reflected the inclusion of a substantial number of $\mathrm{OB} / \mathrm{Gyn}$ hospitals. Between the two rounds, the proportion of outpatients with a prescheduled visit increased remarkably, from $33.5 \%$ to $48.2 \%$, while the referral rate of the inpatients remained unchanged.

\section{Performance on patient experience rating Overall level}

As indicated by the descriptive statistics in figure 2, the proportion of patients who reported a positive experience with their care in the overall ratings varied: on average, in $2018,95 \%$ of patients agreed that they had a good hospitalisation experience, whereas $89 \%$ of patients agreed that they had a good ambulatory care experience.

\section{Ambulatory care}

Table 2A shows the CPEQ-A survey results in 2016 and 18 by domain and hospital categories. With respect to ambulatory care in general, the mean scores of all 
Table 1 Characteristics of respondents from the CPEQ survey in 2016 and 2018

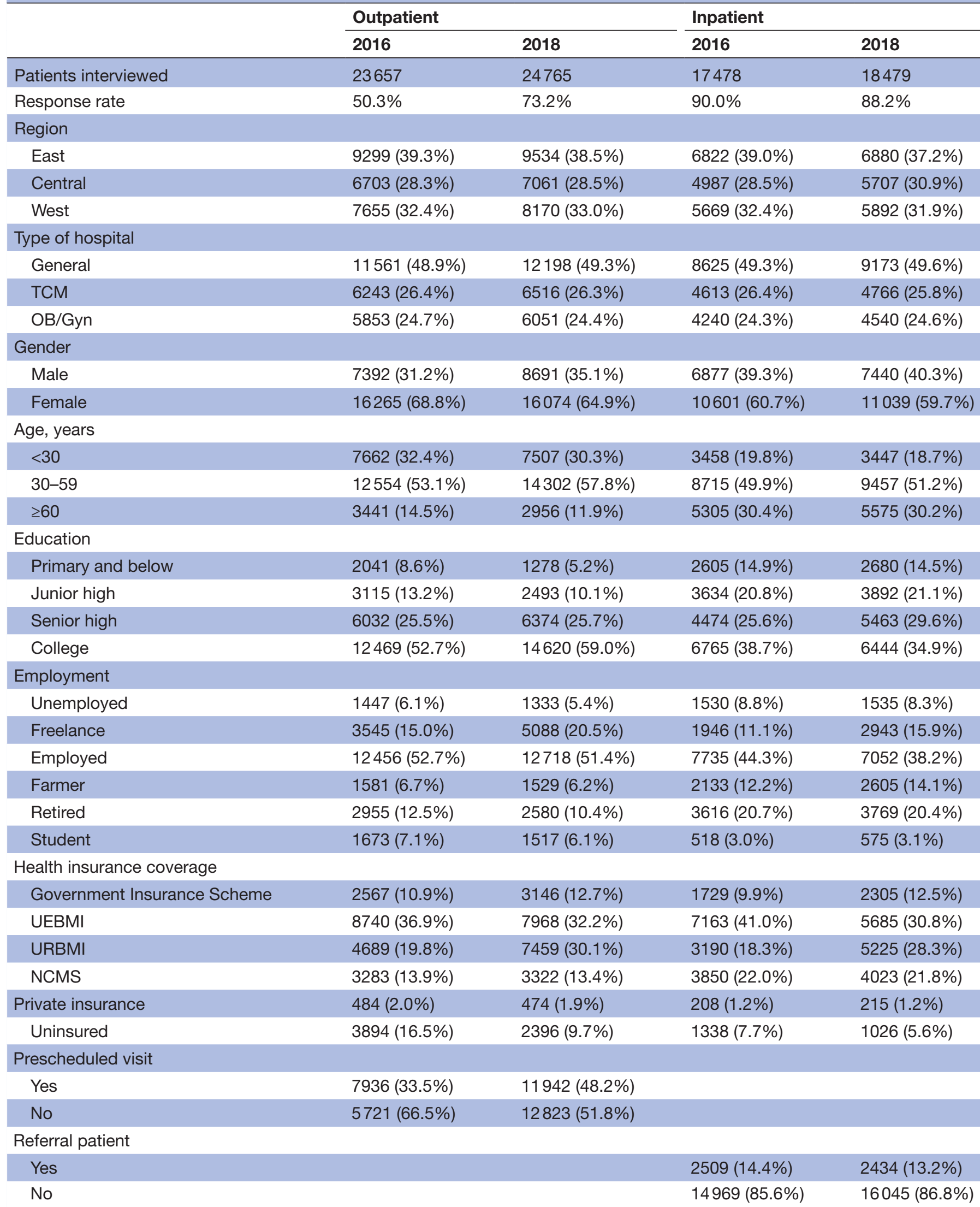

Data are number and \% unless otherwise stated.

NCMS, New Cooperative Medical Scheme; OB/Gyn, obstetrics and gynaecology hospital; TCM, traditional Chinese medicine hospital; UEBMI, Urban Employee Basic Medical Insurance; URBMI, Urban and Rural Resident Basic Medical Insurance. 
2016

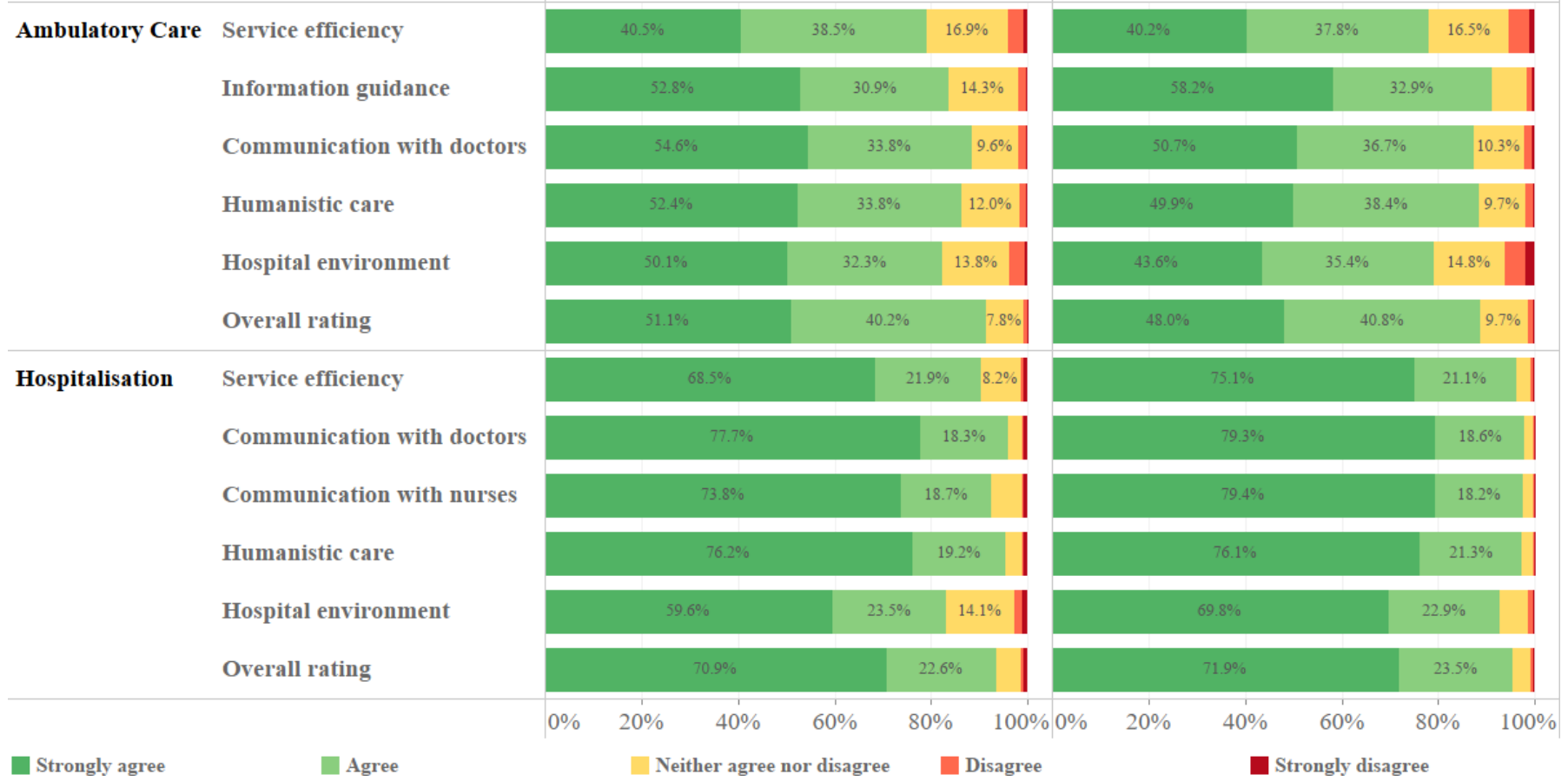

Figure 2 Percentage of patients who reported a positive care experience on specific CPEQ components in 2016 and 2018. CPEQ, Chinese Patient Experience Questionnaire.

domains were over four points both in 2016 and 2018. The dimension of care with the worst performance was service efficiency in 2016 (4.08), and the hospital environment in 2018 (4.05). Changes between the 2016 and 2018 surveys were small, with the largest changes being a 0.12-point improvement in the information guidance score and a 0.17 -point decline in the hospital environment score. Performance in the service efficiency and information guidance domains improved during the initiative $(\mathrm{p}<0.01)$, while performance on another three domains declined $(\mathrm{p}<0.001)$; the performance in the humanistic care domain revealed no significant difference between the baseline and terminal surveys $(p=0.165)$. However, the minimal change in the domains with decreased performance should be interpreted carefully due to the large sample sizes of the study. The distinction between statistical and clinical significance should be considered.

For the general hospitals, performance on service efficiency and information guidance domains were both improved $(\mathrm{p}<0.05)$. With regard to TCM hospitals, only performance in the information guidance domain improved $(\mathrm{p}<0.001)$, while the other domains declined $(\mathrm{p}<0.05)$. For the $\mathrm{OB} / \mathrm{Gyn}$ hospitals, performance in the service efficiency, information guidance and humanistic care domains were improved $(\mathrm{p}<0.05)$, while the hospital environment domain declined $(\mathrm{p}<0.05)$ and received the lowest score across all domains and hospital categories. It is worth noting that this result may reflect the small neglected group of patients with very negative experiences.

\section{Hospitalisation}

Table 2B shows the CPEQ-I survey results in 2016 and 2018 by domain and hospital categories. Performance on most domains improved somewhat between 2016 and $2018(\mathrm{p}<0.001)$. General hospitals performed best in all domains compared with the TCM and OB/Gyn hospitals in 2018. The hospital environment was the lowest rated dimension of care by inpatients across all three hospital categories.

\section{Pooled data analysis}

Putting together patient scores for ambulatory and inpatient care by domain and hospital type, the heatmaps in figures 3 and 4 reveal that performance varied considerably across hospital categories. The patient experience domains were highly correlated overall in both the CPEQ-A (Cronbach's alpha=0.952) and CPEQ-I (Cronbach's alpha $=0.950$ ). Domains with better performance in ambulatory care and hospitalisation were generally reported by patients with higher scores across all subdomain items. Meanwhile, domains with worse performance, such as service efficiency, were also likely to have lower scores for items such as waiting time.

\section{Regression analyses}

With regard to factors affecting the patient experience, our regression analysis revealed two major sets of determinants, including individual characteristics and institutional factors.

We found that female, elderly (60 and above) outpatients with higher education levels (junior high school and above) had a better overall rating of their patient 
Table 2A Domain performance scores on the CPEQ-A in 2016 and 2018, by hospital

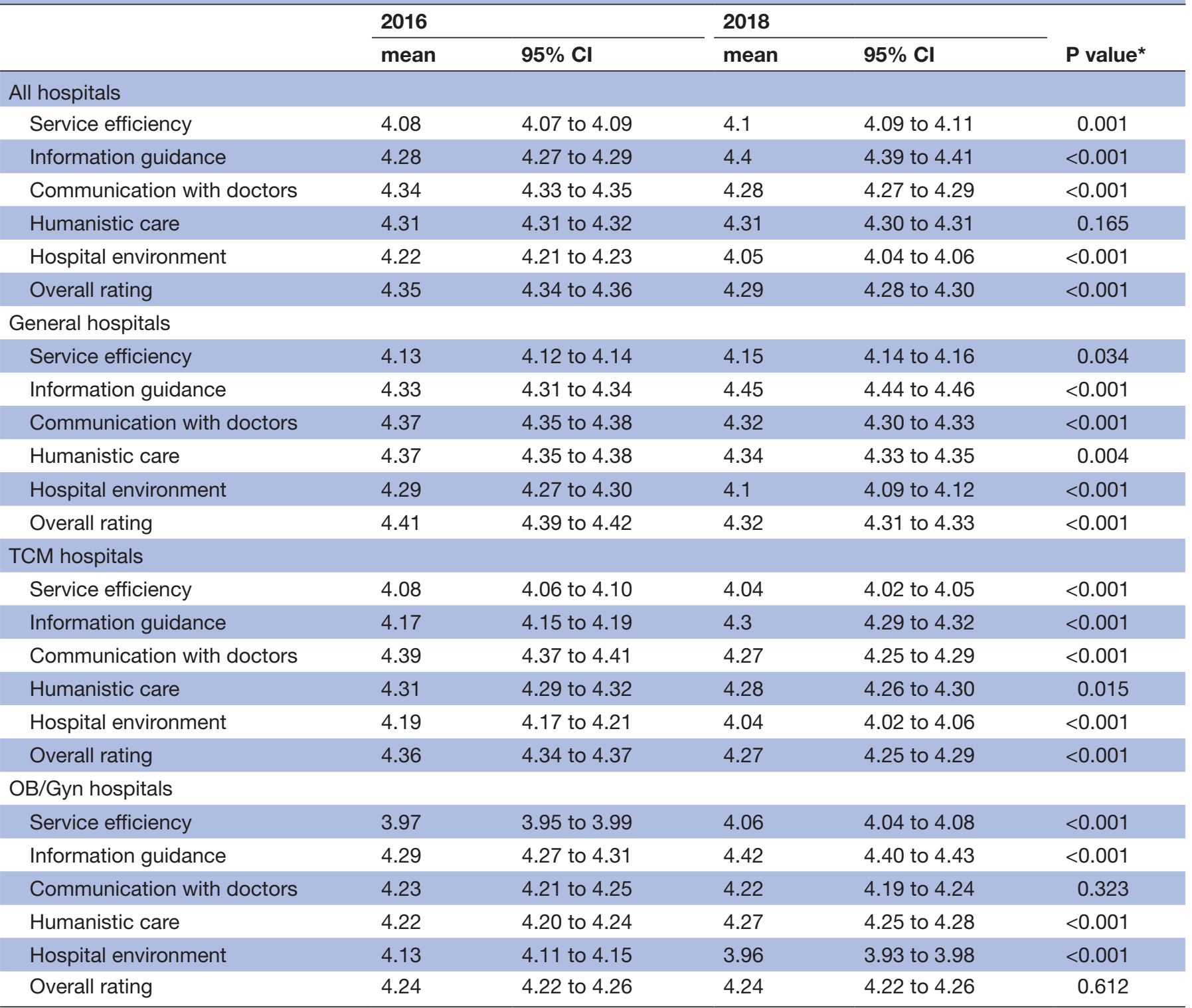

Data are mean and $95 \% \mathrm{Cl}$.

* $P$ values reported for the difference between the 2 years.

CPEQ-A, Chinese Patient Experience Questionnaire for Ambulatory care ; OB/Gyn, obstetrics and gynaecology hospital; TCM, traditional Chinese medicine hospital.

experience. The ratings of the outpatients who were farmers were worse compared with the ratings from patients in all other employment categories $(\mathrm{p}=0.0173)$, while a significant difference was only observed in the general hospital group ( $\mathrm{p}=0.0049)$. Unlike the employment groups in urban areas, who would benefit from a variety of health insurance coverage options, the majority of farmers in China only had the option of New Cooperative Medical Scheme (NCMS) coverage. Correspondingly, we observed that the patients covered by NCMS were also the group with the worst patient experience in all hospital subgroups $(\mathrm{p}<0.0001)$. Furthermore, we found that western hospital outpatients had a worse overall rating of their patient experience, while patients scheduled by appointment had a better perception (table 3A).

We observed that female patients aged 30 years and older with higher education levels (junior high school and above) or patients who were hospitalised in the central hospital region were associated with a better overall rating of the patient experience. The hospitalised patients who were farmers were more likely to report a worse experience in the OB/Gyn hospitals than patients in other employment categories $(\mathrm{p}=0.0002)$. Similar to the results in ambulatory care, the hospitalised patients covered by NCMS also had a less positive patient experience than other health insurance coverage groups. Moreover, we found that referral patients, especially those in 
Table 2B Domain performance scores on the CPEQ-I in 2016 and 2018, by hospital

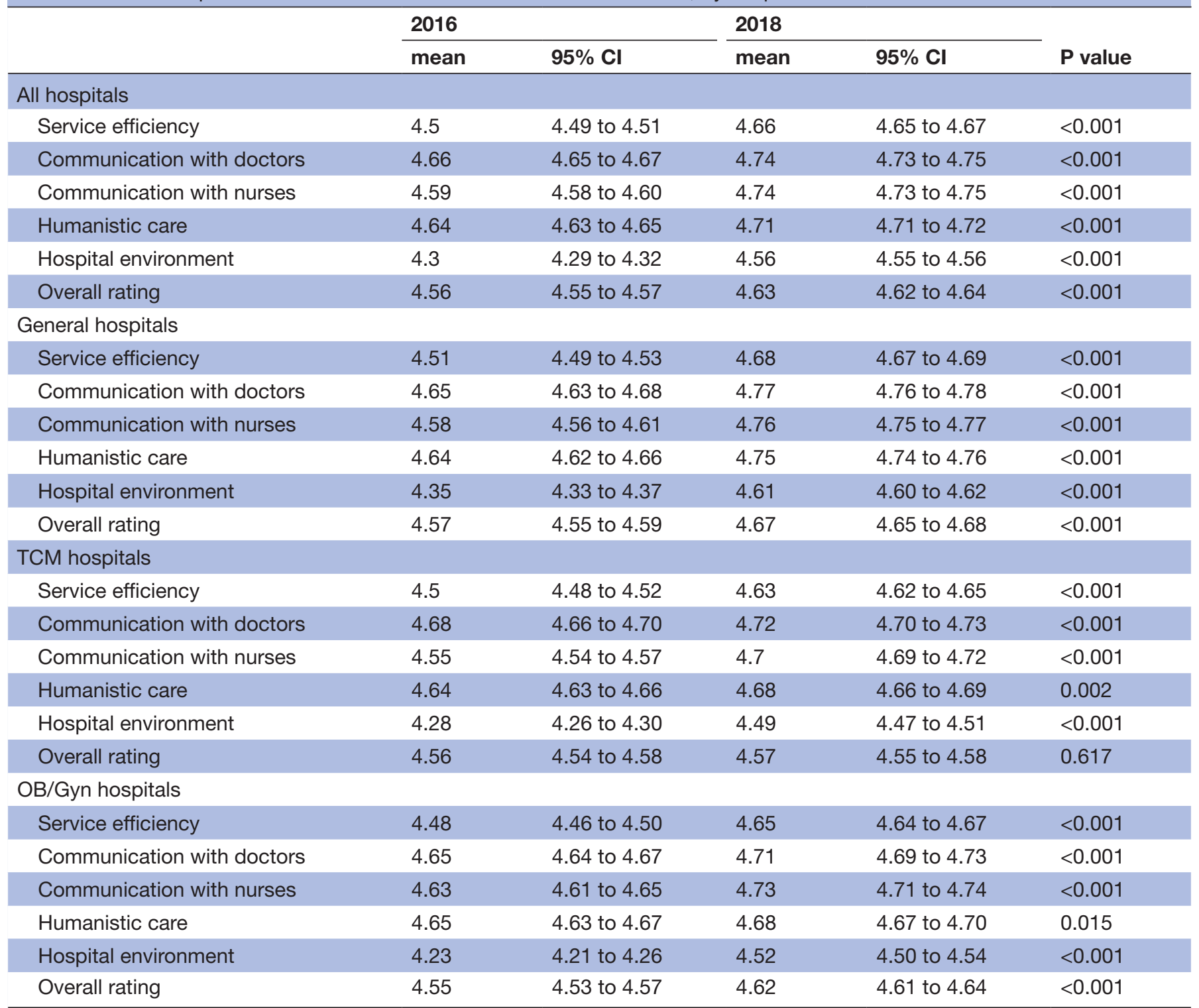

Data are mean and $95 \% \mathrm{Cl}$.

$P$ values reported for the difference between the 2 years.

CPEQ-I, Chinese Patient Experience Questionnaire for Inpatient care; OB/Gyn, obstetrics and gynaecology hospital; TCM, Traditional Chinese medicine hospital.

the $\mathrm{OB} /$ Gyn hospitals, were more likely to have reported a lower overall rating (table $3 \mathrm{~B}$ ).

To further examine the association between overall rating and specific domains of care, we constructed multivariable linear regression models and adjusted for confounding factors, including geographical regions, gender, age, education, employment and health insurance coverage (table 4). We observed that, for each one-point increase in the ratings of the humanistic care, communication with doctors, service efficiency, hospital environment and information guidance domains, the overall rating of the outpatient experience increased by $0.38,0.17,0.15,0.11$ and 0.07 points, respectively. For each one-point increase in the ratings of the humanistic care, communication with doctors, service efficiency, communication with nurses and hospital environment domains, the overall rating of the inpatient experience increased by $0.34,0.21,0.19,0.13$ and 0.11 points, respectively. Humanistic care was the specific aspect of care with the greatest association with the overall patient experience rating, both in the ambulatory care and in the hospitalisation settings.

\section{DISCUSSION}

The CPEQ survey provides insight into the patient experience in China tertiary hospitals since the NHII was launched. For the first time, we generate evidence on the 
Domains of Care

Service efficiency

Information guidance

Communication with doctors

Humanistic care

\section{Hospital environment}

\section{Overall rating}

3.86

Figure 3 Performance scores for the 23 individual items of the CPEQ-A, by hospital category, for both survey years combined The numbers indicate the specific score across items in the CPEQ-A for each hospital category by using the pooled data of the two survey rounds, with the greenest colour indicating the best performance and reddest colour indicating the worst.

CPEQ-A, Chinese Patient Experience Questionnaire for Ambulatory care; TCM, traditional Chinese medicine hospital; OB/Gyn, obstetrics and gynaecology hospital.

quality of care from the perspective of the patient at the national level. We found that patients reported having a somewhat better experience in the hospitalisation setting than in the ambulatory care setting. The general hospitals performed better than TCM and OB/Gyn hospitals on both the CPEQ-A and CPEQ-I surveys. There were significant improvements in the patient hospitalisation experience over the 2016-2018 period, while patients in western hospitals reported less positive experiences than in other regions in both the ambulatory care and hospitalisation settings and across all the hospital categories. Conclusions about the causal relationship between the initiative and improvements in the patient experience cannot be drawn. However, improving the patient experience has been emphasised as the overall goal of the NHII, with explicit phased goals and measures, by the central government of China. The rapid application of mobile internet technology in hospitals, as required by the NHII to enhance public service, is one of the notable examples. Most of the tertiary hospitals in China began to provide web-based appointment services for outpatient visits during the past 3 years. Meanwhile, the waiting time in the hospital decreased as service efficiency improved, which was also observed in our research.

The national portrait of the patient experience in tertiary hospitals, based on the CPEQ survey results, highlighted variations in specific areas of hospital care across different hospital categories and identified explicit priorities for improvement. We found that patients who received ambulatory care generally reported worse experiences in the service efficiency and hospital environment domains than in the other domains. In particular, the outpatient ratings of the hospital environment did not improve much during the initiative, and the hospital environment in the $\mathrm{OB} / \mathrm{Gyn}$ hospitals may have even gotten worse from the perspective of the patient in some extreme cases. The patient experiences of hospitalisation in most dimensions of care, except the hospital environment, were reported to be better than the overall rating of the patient experience. The TCM hospitals performed the worst on the hospital environment domain and improved somewhat during the initiative. These findings suggest that the hospital environment should be the focus for improvement, starting with ambulatory care in the OB/ Gyn hospitals and hospitalisation in the TCM hospitals, in the next phase of the initiative.

Advanced information and communication technology and functional dual referral systems are core action areas for shaping tiered healthcare delivery systems in China, as proposed by the World Bank Group and the WHO. ${ }^{2}$ To align with these actions, the Initiative proposed to promote scheduled appointments for outpatients and a dual-referral system for hospitals by enhancing the utilisation of information technology in service delivery. ${ }^{21}$ Patient responses on the screener question in the CPEQ survey provided the data necessary to monitor the progress 
Domains of Care

Service efficiency
No. Item

Q1 Waiting time before being admitted to a ward was acceptable.

Q2 The admission process was convenient.

Q3 The mechanism for feedback to the hospital was active.

Communication with doctors Q10 Doctors discussed my condition and care patiently.

Q11 Doctors discussed treatment with me patiently.

Q12 I was involved in decisions about my care and treatment.

Communication with nurses

Humanistic care

Hospital environment

Overall rating

\section{Q7}

Q8

The nurses have professional skills.

Q8 The nurses were quick to respond to bells when I called

Q9 The hospital orderly services could meet my needs.

Q13 I was treated with respect and dignity during this hospitalization.

Q14 My privacy has been fully protected during this hospitalization.

Q15 The hospital staff did everything they could to help me with my pain.

Q16 My pain could be well controlled.

Q4 The area around my room was quiet.

Q5 The anti-slip facilities were well equipped.

Q6 The hospital food supply could meet my needs.

Q17 The medical bill was easy to understand.

Q18 I had a good hospitalization experience in this hospital.

Q19 I would recommend this hospital to my friends and family.

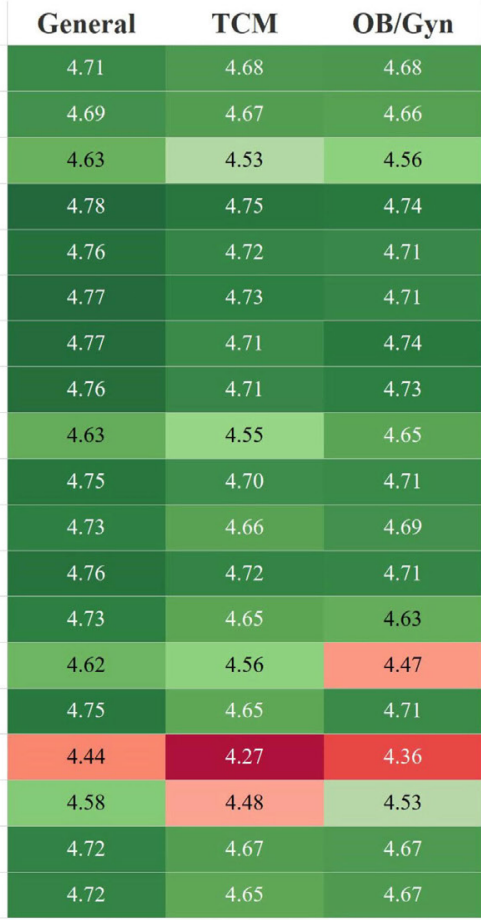

4.78

4.27

Figure 4 Performance scores for the 19 individual items of the CPEQ-I, by hospital category, for both survey years combined. The numbers indicate the specific score across items in the CPEQ-I for each hospital category by using the pooled data of the two survey rounds, with the greenest colour indicating the best performance and reddest colour indicating the worst. CPEQ-I, Chinese Patient Experience Questionnaire for Inpatient care; TCM, traditional Chinese medicine hospital; OB/Gyn, obstetrics and gynaecology hospital.

of these measures towards improving the patient experience. The proportion of outpatients who had a scheduled an appointment for ambulatory care increased from $33.5 \%$ in 2016 to $48.2 \%$ in 2018 , with a higher overall patient experience rating across all hospital categories than those without an appointment. Meanwhile, the proportion of referral patient in the terminal survey did not change too much from the baseline level, and referral patients were associated with a lower overall rating of the patient experience in all hospitals and in the hospital subgroups. These results offer preliminary evidence that the Initiative may contribute to better patient experience of ambulatory care provide by hospitals that have adopted measures in appointment service; there was room for improvement for referral patients as well as in the dual-referral system.

Universal health insurance coverage has been nearly achieved in China since the launch of the national reform in 2009, with the introduction of several health insurance plans with different parallel compensation levels that have been established for various groups of residents. ${ }^{2} \mathrm{We}$ found that farmers reported the worst experiences in the overall rating of the patient experience in both the ambulatory care and hospitalisation setting. Given that most of the farmers were enrolled in the New Cooperative Medical Scheme (NCMS), this result was in accordance with variations in the patient experience among the different insurance coverage options, with NCMS patient ratings that were worse than ratings from patients covered by other insurance. Previous research, based on the 2011 and 2015 surveys of the China Health and Retirement Longitudinal Study, reported that URRMI (integration of URBMI and NCMS) has a limited effect on healthcare utilisation by residents in the pilot provinces ${ }^{31}$; we further identified the tangible gaps in the patient perspective of care delivery among the various insurance coverage options. These gaps are likely attributable to disparities in the benefit packages among various health insurance plans $^{32}$ and to inequities in the quality of life of patients under the plans. ${ }^{33}$ Within the context of ongoing national reform to integrate the fragmented insurance system of China, our results emphasise that equal quality of patient care is a critical entitlement for all residents.

In line with previous studies on the British, ${ }^{9} 1634$ American $^{35} 36$ and Canadian patient experience, ${ }^{10}$ we found that the individual sociodemographic characteristics of the patients in China are systematically related to their overall rating of the patient experience. As a result, we adjusted our analysis, considering the specific characteristics of the patient as confounders, to examine the association between the overall rating and the specific aspects of patient services. We identified that the provider's performance in the humanistic care domain has the largest positive effect on the overall rating of the patient experience in both the ambulatory care and hospitalisation setting and across all hospital categories. However, Kemp et al 


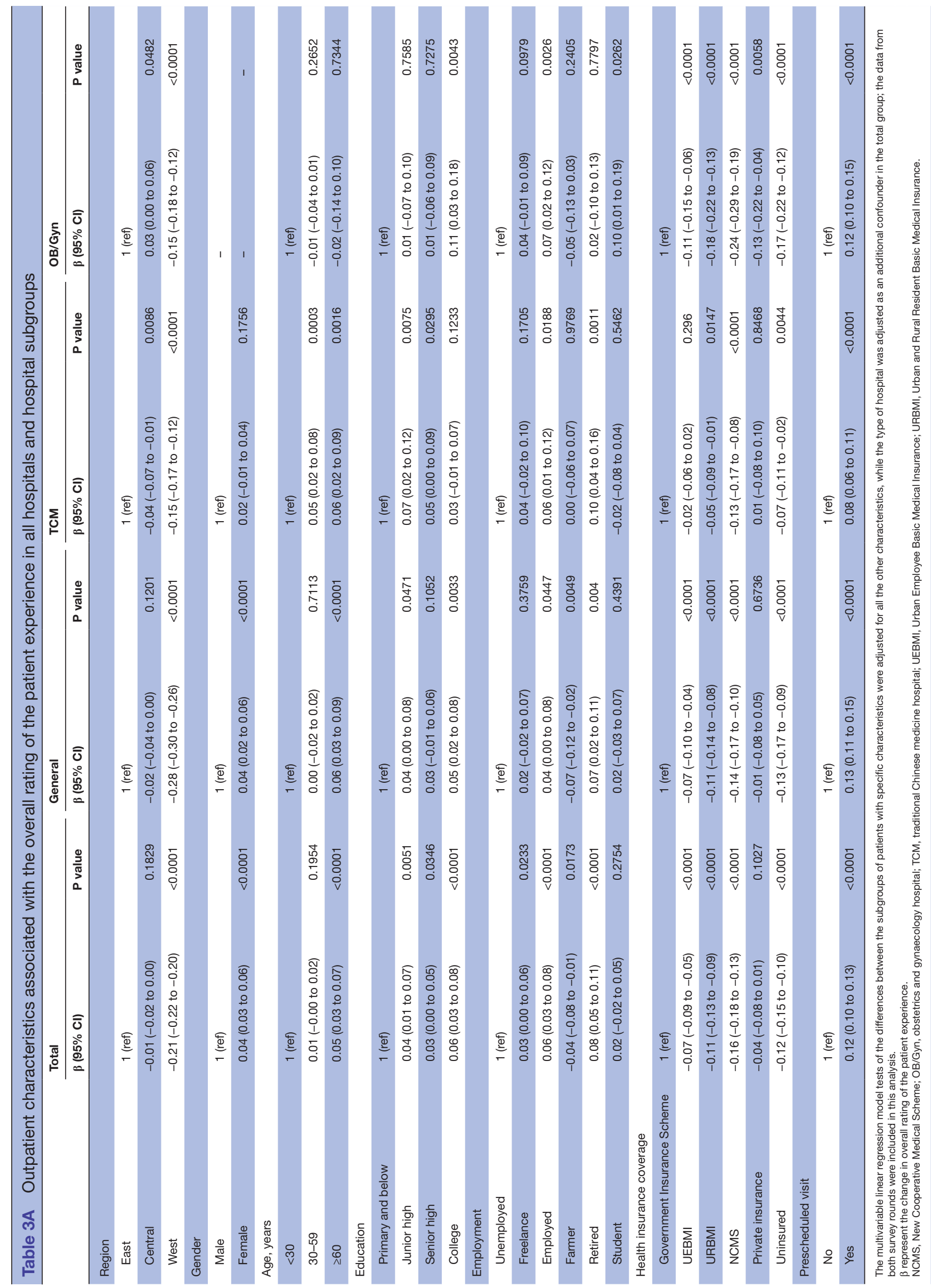




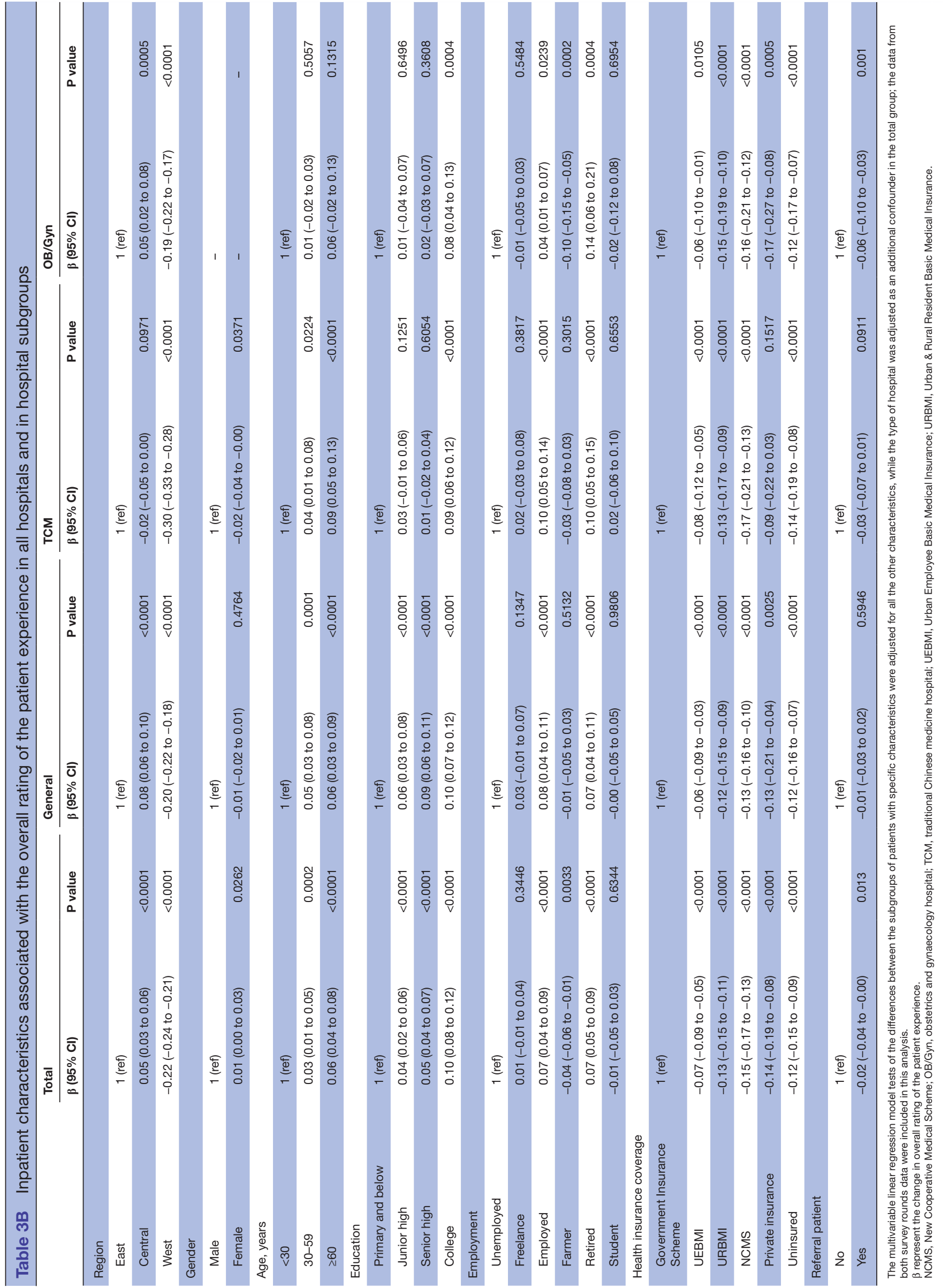




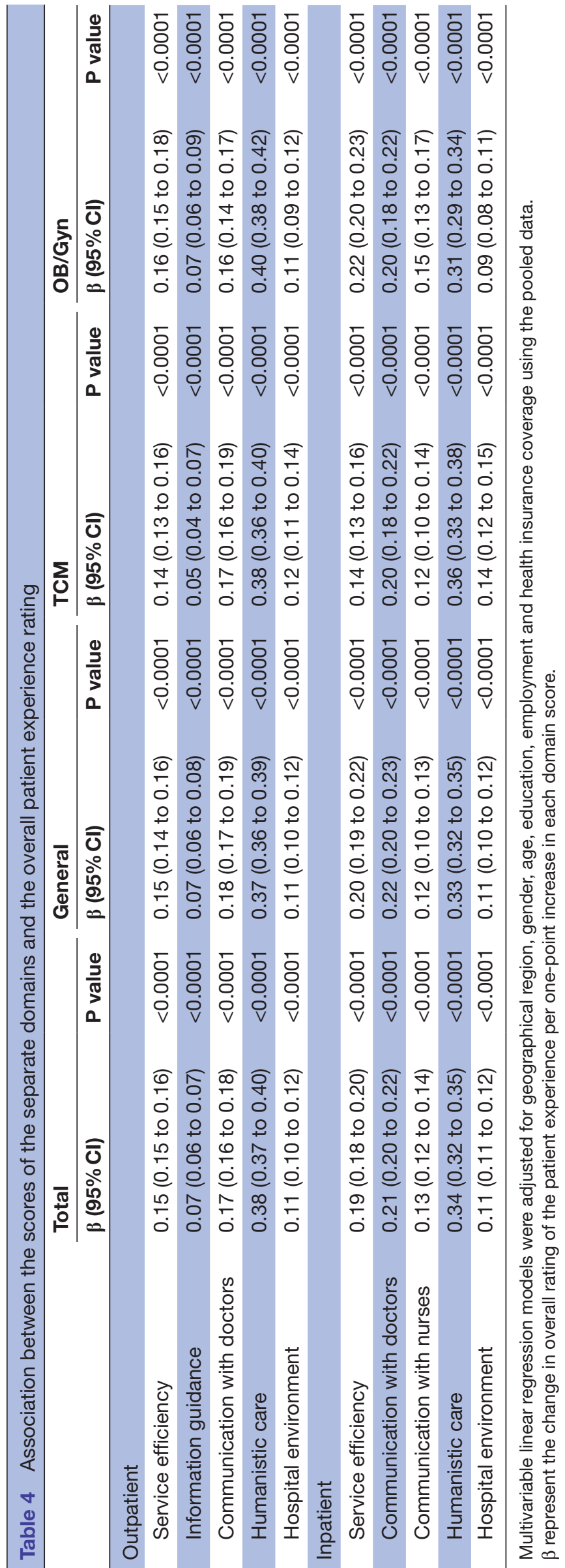

found that communication with nurses has the strongest correlation with the overall inpatient experience based on the HCAHPS survey in the Canadian context. ${ }^{37}$ Aaronson et al reported that communication mattered most for the overall emergency department rating in a retrospective cohort study in America. ${ }^{38}$ Sipsma et al also indicated that communication with nurses was most strongly associated with overall inpatient ratings in seven rural provinces in China. ${ }^{26}$ These findings highlight that the variation in priority areas may contribute to improving the patient experience in different regions and populations, whereas both studies enhance the consensus among providers that interpersonal-related initiatives have more substantial roles in the improvement of the patient experience, rather than physical attributes of institutions. ${ }^{37}$

Our study has several limitations. First, we collected data from 117 tertiary hospitals, across 31 PMAs in China, that are not nationally representative of the three levels of Chinese hospitals (level I-III with a higher level representing the larger size and higher quality of facilities). Second, all the surveys were administered during the hospital visit or hospitalisation by face-to-face interview; although we set the patient inclusion criteria to be those who would be ready to leave or be discharged from hospital, there was still the possibility that the inpatients were unable to provide truthful answers. Third, $550 \%$ of outpatients rejected or failed to complete the first round of the survey. Although we enhanced the patient recruitment for the second round of the survey by improving the interviewers' communication skills, the response rate only improved to $73.2 \%$; therefore, the non-response bias might affect the reliability of our measures. Previously, researchers noted that patients who had a less positive care experience were also less likely to respond, resulting in a non-response bias of the score; this pattern might be greater in hospitals with lower response rates. ${ }^{39}$ Although the response rates in our study were acceptable ${ }^{5}$ non-response weighting should be considered in future work. Fourth, we presented our survey results without case-mix adjustment, and this issue should be carefully examined in future studies. Finally, the two rounds of CPEQ surveys were conducted in the month of January, just before the Chinese Spring Festival. This might have an impact on the utilisation rate of both outpatient and inpatient care services. Future surveys might want to examine seasonal variations.

\section{CONCLUSIONS}

To our knowledge, this study is the first to present an overview of the patient experience in Chinese tertiary hospitals. China's NHII, launched in 2015, seems to have had a positive impact, as indicated by the steady, although unremarkable, increase of the patient experience scores in many domains over the 2016-2018 period. Chinese patients generally rated the clinical aspects of care positively but rated the environmental, interpersonal and social services aspects of care less positively. While the 
overall patient experience was found to be positive and encouraging, areas for further improvements, such as environmental and humanistic aspects of care and non-medical services, were highlighted. In particular, the farmers and the users of TCM services are the groups of people whose experiences deserve special policy attention.

As the pillar of healthcare quality, the patient experience can be utilised as a standardised performance measure in monitoring the progress of the national initiative on quality improvement, and should also be included in health system performance assessment. To realise government commitment to health reform for the population, independent performance assessment of the patient experience and public reporting mechanisms in the domestic context are essential in China as well as in other transitioning health systems.

\section{Author affiliations}

${ }^{1}$ Institute of Medical Information/Center for Health Policy and Management, Chinese Academy of Medical Sciences and Peking Union Medical College, Beijing, China ${ }^{2}$ School of Public Health, Chinese Academy of Medical Sciences and Peking Union Medical College, Beijing, China

${ }^{3}$ Peking University International Hospital, Beijing, China

${ }^{4}$ National Institute of Hospital Administration, Beijing, China

${ }^{5}$ School of Public Health, Fudan University, Shanghai, China

${ }^{6}$ School of Public Policy and Administration, Xi'an Jiaotong University, Xi'an, China ${ }^{7}$ Department of Health Policy and Management, Fielding School of Public Health, UCLA, Los Angeles, California, USA

${ }^{8}$ Department of Population Medicine, Harvard Pilgrim Health Care Institute, Harvard Medical School, Boston, Massachusetts, USA

Acknowledgements We acknowledge Dr Yahui Jiao from the National Health Commission for her outstanding administrative support in the project execution. We appreciate the contributions made by the leading experts of all regional steering committees, they are Fengmei Jiang (Beijing Hospital Association), Yunling Zhang (China Association of Chinese Medicine), Jun Ma (Tianjin Medical University), Yunzhang Liu (Hebei Medical University), Huazhang Wu (China Medical University), Dongfu Qian (Nanjing Medical University), Xiaolin Luo (Zhejiang Medical Quality Control Committee), Qiaoyu Jiang (Fujian Medical University), Shixue Li (Shandong University), Yong Zhang (Southern Medical University), Peng Wu (Hainan Medical Quality Control Committee), Changjiu Zhao (Harbin Medical University), Zhuang Cao (Jilin Medical Quality Monitoring and Evaluation Center), Yan Hou (Shanxi Hospital Association), Qicheng Jiang (Anhui Medical University), Xiaojun Zhou (Nanchang University), Xinkui Liu (Zhengzhou University), Zhiguo Zhang (Huazhong University of Science and Technology), Tubao Yang (Central South University), Jinlong Wu (Inner Mongolia Physicians Association), Guo Zhang (The People's Hospital of Guangxi Zhuang Autonomous Region), Hong Zhou (Chongqing Cancer Hospital), Xiaohua Zheng (Sichuang Academy of Medical Sciences), Hong Zheng (Zunyi Medical University), Ping Fan (Yunnan Hospital Association), Yong Jiang (Tibet University Medical College), Jinlin Liu (Xi'an Jiaotong University), Kehu Yang (Lanzhou University), Xiaoming Ma (Qinghai Medical Association), Yuhong Zhang (Ningxia Medical University) and Hua Yao (Xinjiang Medical University). Thanks for them coordinating regional experts, organising interviewer training and supervising the survey during the field study, which helps the project investigation complete timely. And we are grateful for all the hospitals and patients for their participation and active cooperation in the project.

Contributors Conceptualisation: YL. Data curation: GH, YC, QL, SW, JG, SL, ZW and PZ. Formal analysis: GH. Funding acquisition: YL and GH. Investigation: GH, YC, QL, SW, JG, SL, ZW, PZ, JS and LH. Methodology: YL and GH. Project administration: GH, JS, LH and HZ. Resources: LL, YM and JM. Software: GH. Supervision: JM and YL. Visualization: GH. Writing —original draft: GH, YC, QL and SL. Writing—review and editing: JN, JM and YL.

Funding This research was funded by the Bureau of Medical Administration of National Health Commission, PR China; the Non-profit Central Research Institute Fund of Chinese Academy of Medical Sciences (2018PT33009) and the Special Research Fund for Central Universities, Peking Union Medical College (3332019087).
Competing interests None declared.

\section{Patient consent for publication Obtained.}

Ethics approval The study was approved by the institutional review board of the Peking Union Medical College (71532014). All respondents provided their oral consent to participate in the survey before the interview.

Provenance and peer review Not commissioned; externally peer reviewed.

Data availability statement Data are available upon reasonable request.

Open access This is an open access article distributed in accordance with the Creative Commons Attribution Non Commercial (CC BY-NC 4.0) license, which permits others to distribute, remix, adapt, build upon this work non-commercially, and license their derivative works on different terms, provided the original work is properly cited, appropriate credit is given, any changes made indicated, and the use is non-commercial. See: http://creativecommons.org/licenses/by-nc/4.0/.

\section{REFERENCES}

1. Liu Y, Kong Q, Yuan S, et al. Factors influencing choice of health system access level in China: a systematic review. PLoS One 2018;13:e0201887.

2. World Bank and World Health Organization. Healthy China: deepening health reform in China: building high-quality and valuebased service delivery. Washington, DC: World Bank Publications, 2019. ISBN: 978-1-4648-1323-8. https://elibrary.worldbank.org/doi/ book/10.1596/978-1-4648-1263-7

3. Zhou M, Zhao L, Campy KS, et al. Changing of China's health policy and Doctor-Patient relationship: 1949-2016. Health Policy and Technology 2017;6:358-67.

4. Black N, Jenkinson C. Measuring patients' experiences and outcomes. BMJ 2009;339:b2495.

5. Ahmed F, Burt J, Roland M. Measuring patient experience: concepts and methods. Patient 2014;7:235-41.

6. Wolf JA, Niederhauser V, Marshburn D, et al. Defining patient experience. Patient Experience Journal 2014;1:7-19.

7. Jenkinson C, Coulter A, Bruster S. Patients' experiences and satisfaction with health care: results of a questionnaire study of specific aspects of care. Qual Saf Health Care 2002;11:335-9.

8. Bleich S, Ozaltin E, Murray CK. How does satisfaction with the health-care system relate to patient experience? Bull World Health Organ 2009;87:271-8.

9. Salisbury C, Wallace M, Montgomery AA. Patients' experience and satisfaction in primary care: secondary analysis using multilevel modelling. BMJ 2010;341:c5004.

10. Kemp KA, Santana MJ, Southern DA, et al. Association of inpatient Hospital experience with patient safety indicators: a cross-sectional, Canadian study. BMJ Open 2016;6:e11242.

11. Goldstein E, Farquhar M, Crofton C, et al. Measuring hospital care from the patients' perspective: an overview of the CAHPS $®$ Hospital survey development process. Health Serv Res 2005;40:1977-95.

12. Giordano LA, Elliott MN, Goldstein E, et al. Development, implementation, and public reporting of the HCAHPS survey. Med Care Res Rev 2010;67:27-37.

13. Breckenridge K, Bekker HL, Gibbons E, et al. How to routinely collect data on patient-reported outcome and experience measures in renal registries in Europe: an expert consensus meeting. Nephrol. Dial. Transplant. 2015;30:1605-14.

14. Wiig S, Storm M, Aase K, et al. Investigating the use of patient involvement and patient experience in quality improvement in Norway: rhetoric or reality? BMC Health Serv Res 2013;13:206.

15. DeCourcy A, West E, Barron D. The National adult inpatient survey conducted in the English National health service from 2002 to 2009 : how have the data been used and what do we know as a result? BMC Health Serv Res 2012;12:71.

16. Paddison C, Elliott M, Parker R, et al. Should measures of patient experience in primary care be adjusted for case mix? Evidence from the English general practice patient survey. BMJ Qual Saf 2012;21:634-40.

17. Squires A, Bruyneel L, Aiken LH, et al. Cross-Cultural evaluation of the relevance of the HCAHPS survey in five European countries. International Journal for Quality in Health Care 2012;24:470-5.

18. Robert G, Cornwell J. Rethinking policy approaches to measuring and improving patient experience. $J$ Health Serv Res Policy 2013;18:67-9.

19. Tefera L, Lehrman WG, Conway P. Measurement of the patient experience: Clarifying facts, myths, and approaches. JAMA 2016;315:2167-8. 
20. Wells S, Tamir O, Gray J, et al. Are quality improvement Collaboratives effective? A systematic review. BMJ Qual Saf 2018;27:226-40.

21. National Health and Family Planning Commission. Announcement of implementing the National healthcare improvement initiative, 2015. Available: http://www.nhc.gov.cn/yzygj/s3593g/201501/5584853c fa254d1aa4e38de0700891fa.shtml [Accessed 20190301].

22. National Health and Family Planning Commission. Announcement of implementation strategy of the National Healthcare Improvement Initiative(2015-2017), 2015. Available: http://www.nhc.gov.cn/yzygj/ s3593g/201506/cbe5494282d24e97a001e81e0325e1b1.shtml [Accessed 20190301].

23. Wang X, Jiang R, Li J, et al. What do patients care most about in China's public hospitals? Interviews with patients in Jiangsu Province. BMC Health Serv Res 2018;18.

24. Lu C, Hu Y, Xie J, et al. The use of mobile health applications to improve patient experience: cross-sectional study in Chinese public hospitals. JMIR Mhealth Uhealth 2018;6:e126.

25. Bao Y, Fan G, Zou D, et al. Patient experience with outpatient encounters at public hospitals in Shanghai: examining different aspects of physician services and implications of overcrowding. PLoS One 2017;12:e171684.

26. Sipsma H, Liu Y, Wang H, et al. Patient experiences with inpatient care in rural China. International Journal for Quality in Health Care 2013;25:452-8.

27. Wong ELY, Leung MCM, Cheung AWL, et al. A population-based survey using PPE-15: relationship of care aspects to patient satisfaction in Hong Kong. Int J Qual Health C 2011;23:390-6.

28. Liu C, Wu Y, Chi X. Relationship preferences and experience of primary care patients in continuity of care: a case study in Beijing, China. BMC Health Serv Res 2017;17:585.

29. Hu G. Study on patient experience and public opinion of healthcare in China. PhD. Beijing: Peking Union Medical College, 2018.

30. Jha AK, Orav EJ, Zheng J, et al. Patients' perception of hospital care in the United States. N Engl J Med Overseas Ed 2008;359:1921-31.
31. Su D, Chen Y-chun, Gao H-xia, et al. Effect of integrated urban and rural residents medical insurance on the utilisation of medical services by residents in China: a propensity score matching with difference-in-differences regression approach. BMJ Open 2019;9:e26408

32. Meng Q, Fang H, Liu X, et al. Consolidating the social health insurance schemes in China: towards an equitable and efficient health system. The Lancet 2015;386:1484-92.

33. Su M, Zhou Z, Si Y, et al. Comparing the effects of China's three basic health insurance schemes on the equity of health-related quality of life: using the method of coarsened exact matching. Health Qual Life Outcomes 2018;16.

34. Mead N, Roland M. Understanding why some ethnic minority patients evaluate medical care more negatively than white patients: a cross sectional analysis of a routine patient survey in English general practices. BMJ 2009;339:b3450.

35. Chou W-YS, Wang LC, Finney Rutten LJ, et al. Factors associated with Americans' ratings of health care quality: what do they tell us about the raters and the health care system? J Health Commun 2010;15:147-56.

36. Zhu J, Weingart SN, Ritter GA, et al. Racial/Ethnic disparities in patient experience with communication in hospitals: real differences or measurement errors? Med Care 2015;53:446-54.

37. Kemp K, McCormack B, Chan N, et al. Correlation of inpatient experience survey items and domains with overall Hospital rating. Journal of Patient Experience 2015;2:29-36.

38. Aaronson EL, Mort E, Sonis JD, et al. Overall emergency department rating: identifying the factors that matter most to patient experience. $J$ Healthc Qual 2018;40:367-76.

39. Elliott MN, Zaslavsky AM, Goldstein E, et al. Effects of Survey Mode, Patient Mix, and Nonresponse on CAHPS ${ }^{\circledR}$ Hospital Survey Scores. Health Serv Res 2009;44:501-18. 